\title{
Understanding electron flow in conducting polymer films: injection, mobility, recombination and mesostructure
}

\author{
A M Stoneham ${ }^{1}$, M M D Ramos' ${ }^{2}$ A M Almeida' 2 H M G Correia ${ }^{2}$, \\ R M Ribeiro ${ }^{2}$, H Ness ${ }^{3}$ and A J Fisher ${ }^{1}$ \\ ${ }^{1}$ Department of Physics and Astronomy, University College London, Gower Street, \\ London WC1E 6BT, UK \\ 2 Departamento de Fisica, Universidade do Minho, Braga 4710-057, Portugal \\ ${ }^{3}$ CEA-Saclay, DSM/DRECAM/SPCSI, Bât. 462, F-91191 Gif sur Yvette, France
}

Received 12 August 2002

Published 11 October 2002

Online at stacks.iop.org/JPhysCM/14/9877

\begin{abstract}
We survey the current state of models for electronic processes in conducting polymer devices, especially light-emitting diodes. We pay special attention to several processes that have been somewhat neglected in the previous literature: charge injection from electrodes into a polymer sample, mobility of charge-or energy-carrying defects within a single molecule and (more briefly) transfer of carriers between molecules and the interaction between the charge transport and the mesostructure of the polymer. Within all these areas substantial progress has been made in recent years in elucidating the important physics, but further progress is needed to make quantitative contact with experiment.
\end{abstract}

\section{Introduction}

In some respects, conducting polymers mimic traditional semiconductors, like silicon or the III-Vs. In particular, they are already the basis of effective light-emitting diodes (LEDs) (Burroughes et al 1990, Friend et al 1999), and promise a wider range of successful devices. Does it make sense to regard a thin film of polymer in the same way as a slab of conventional semiconductor of the same dimensions, band gap and carrier mobility? There are two major reasons for moving to other descriptions. First, the nature of the electronic excitation in polymers is different, partly because of the relatively large electron-phonon coupling. This coupling has profound effects on carrier injection and on carrier transfer between strands (a term we shall use informally to describe the individual conjugated segments of the molecules or, in the case of branched or cross-linked molecules, a component bounded by cross-links or branch points). Secondly, the structures of semiconducting polymers do not have the simplicity of crystals like $\mathrm{Si}$, but have texture. The polymer strands, relatively short in comparison with conventional industrial polymers, typically have less than 10 monomer units. However, the 


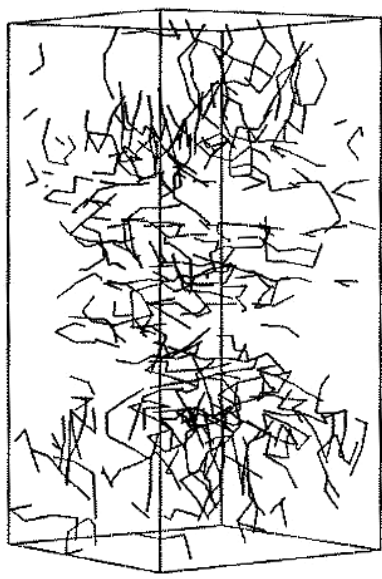

Figure 1. An illustration of the polymer mesostructure from a 'typical' simulation.

number of units is not controlled exactly, and the strands may be bent or twisted. There may be some branching and cross-linking. The axes of the polymer strands can be controlled only incompletely, and indeed the topology of the system may be significant. This 'spaghetti structure' has important consequences; a feel for its complexity can be obtained by examining figure 1, showing a 'typical' mesostructure from one of our simulations.

In the present paper, we shall discuss the modelling of semiconductor polymer devices, emphasizing the effects of electron-phonon coupling and of mesostructure. We shall do so by following the several steps by which a carrier can be injected and move through the device. However, for simplicity, we shall omit discussion of some features that, while important for the functioning of particular devices, would further complicate the picture. In particular, we will not discuss electron- or hole-barrier layers, nor the optical characteristics of the light-emitting layers (which are non-trivial since their thickness is less than the wavelength of light-(Kim et al 2000, Bolognesi et al 2001)). Our aim is to understand the roles of electron-phonon coupling and of texture, so as to make effective modelling possible.

For conventional crystalline semiconductors, like the III-Vs, optical properties are controlled by band-gap engineering: alloying to adjust the band edges with minimal effects on the lattice parameter. Many devices can be described successfully in terms of an isotropic homogeneous continuum model, using a standard semiconductor picture for properties such as carrier density and mobility. In most cases, space charge can be described by the Poisson equation for a consistently chosen continuum charge density.

For organic semiconductors, the situation is quite different. Some of the important features are determined largely by the individual molecular strands (e.g., intra-molecular mobility; band-edge positions; importance of non-radiative recombination). Other features (e.g., isotropy of the macroscopic mobility; propensity to charge trapping) depend strongly on the connectivity and texture (including both the 'spaghetti structure' and possible inclusions or voids). There is considerable experimental evidence that the texture is important (Bloor et al 1975, Friend et al 1999, Gao et al 1999, Nguyen et al 2000, Shi et al 2000, Whitehead et al 2000). Further features are important for electron transfer between an electrode and a molecule, or between molecules within the film.

It is not trivial to improve the performance of a device based on semiconducting polymers, since the various factors are interdependent. There are four broad classes of challenge (Stoneham and Ramos 2001). The first is at the molecular level: how can one identify and model a molecule with the right electronic properties? Much of the progress has been made 
through the chemical insight of those who have synthesized the molecules. Self-consistent molecular dynamics (Wallace et al 1991a, 1991b) is an additional tool (see section 3), which allows questions to be answered that are difficult by direct experimental observation. The second challenge concerns the identification of an acceptable production process, including integration with other materials. A third challenge lies at the microstructural level: how best can the ensemble of polymer molecules be organized to optimize performance for some application? This challenge (see also Ramos et al 1994, Ramos and Stoneham2000a, 2000b) is analysed in section 5. There would seem to be considerable scope for control and optimization, especially if the texture can be controlled within the film. Moreover, the compromises between trapping, transport across the film, radiative recombination and non-radiative recombination are not always intuitive (Stoneham and Ramos 2001). Optimization is even more subtle when there are inclusions within the polymer film. The differences between steady-state and pulsed operation suggest that even relatively simple calculations are of value. The final challenge, which we shall not discuss, concerns how best to integrate macroscopic components (films, wires) for some application, whether electroluminescence, screening, sensors or solar panels. This integration would involve factors such as the electromagnetic boundary conditions that constrain the electric field to be normal to the surface of a conductor. We also neglect nonradiative transitions arising from energy transfer from the excited organic strand to the metal (Hochfilzer et al 1998).

The several physical processes might be modelled in a number of possible ways. Our approach, which may broadly be illustrated by the schematic diagram shown below, describes just one of these possible mesoscopic descriptions as an illustration of this class of methods. In this paper, we shall discuss calculations for components 1-4 and their implications for property predictions 6 . The overall plan of the paper is to follow the fate of an injected carrier as it moves through the film over successively larger length scales. First, we consider the physics at the molecular level, starting with the question: what determines the probability that electrons of different energies can be injected from an electrode into a polymer (section 2)? Next, we examine some features of the motion of a carrier within a chain, including the mobility and the dependence of key quantities such as the ionization energy and electron affinity on the chain length and curvature (section 3). In section 4 we discuss inter-chain transport, before moving on to describe some of the effects of the mesoscopic structure of the films in section 5 .

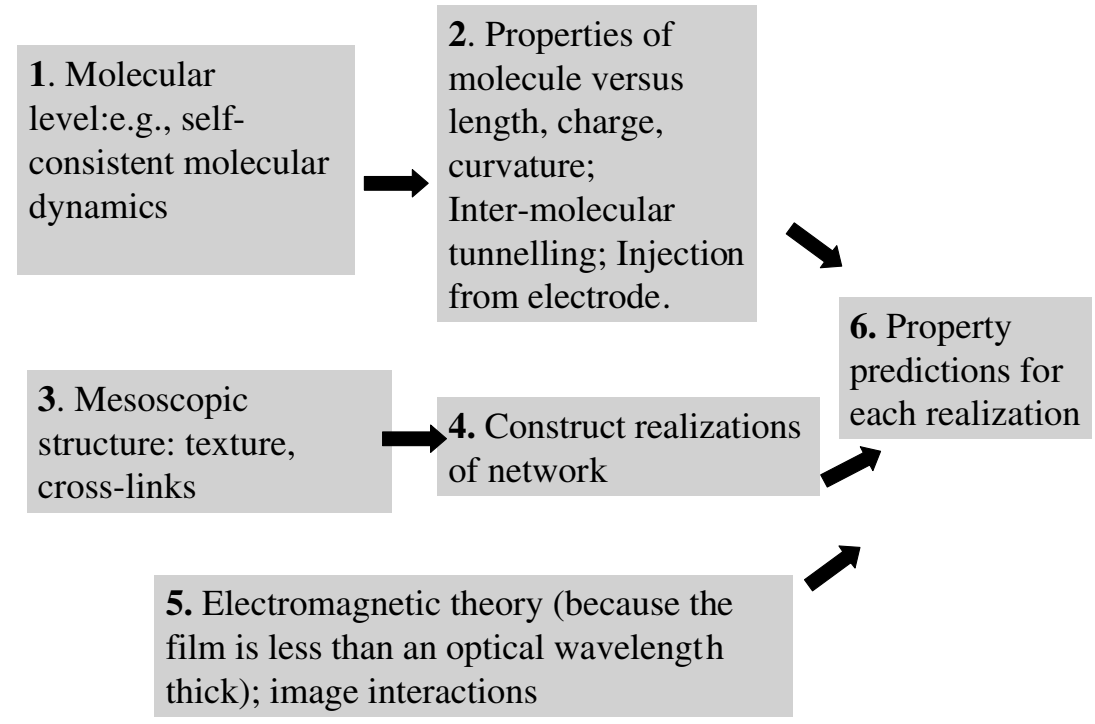




\section{Injection and ejection: electrode to chain}

There have been a number of previous treatments of various aspects of the carrier injection process. For example, Conwell and Wu (1997) treat a simple model of the injection process, involving tunnelling into a statistical distribution of pre-existing polaron states. The mean energy of this distribution is determined by a combination of

(a) the polaron binding energy relative to the band edge,

(b) the applied electric field and

(c) the image interaction with the electrodes.

In a contrasting approach, other groups have calculated the atomic and electronic structure of a few particular realizations of the polymer-metal interface in considerable detail. Notably, Curioni and Andreoni (2000, 2001) performed ab initio molecular dynamics calculations on $\mathrm{Alq}_{3}$ (a relatively small molecule by the standards of polymer electronics) adjacent to $\mathrm{Li}$, $\mathrm{Al}$ and $\mathrm{Ca}$ surfaces. Since these simulations contain a very accurate description of the local chemical bonding they can show up differences in behaviour between different metals very effectively, and in particular show up the limitations of models of the work function that ignore the local atomic structure of the interface. However, because of the computational resources required, only relatively small systems and relatively short times (a few picoseconds) can be treated. Furthermore, because the electronic structure method works on the ground state potential surface, it does not give direct information about the charge injection process.

Our aim, in the calculations described here, is to complement these approaches by including a key further ingredient in the problem: the fact that the process of forming the polaron is a dynamical one. In other words, the polaron state which the carrier will occupy is not present until the electron is there to create it, by means of the forces it exerts on the atoms in the molecule. In order to capture this new piece of physics we will temporarily ignore other important aspects of the problem, including the true three-dimensional chemical structure of the interface, with the awareness that they must be re-introduced later once the fundamental step of polaron formation is understood.

\subsection{Model of coherent injection}

We first discuss how we model transport in the coherent limit, i.e. when the electron and the atomic vibrations with which it interacts participate in a single, coherent, quantum-mechanical process. This is only true for relatively small individual parts of the transport process; in this paper, we apply this model primarily to the injection of charge into a molecule, but in our previous work (Ness and Fisher 1999, 2002a, 2002b, Ness et al 2001) we also considered a transport process that was entirely coherent throughout a single molecule. Such a coherent process corresponds to an idealized 'molecular electronics' experiment, rather than to transport in a real conducting polymer film.

When the molecule is connected to the metallic electrode, we assume that the corresponding Hamiltonian matrix elements are such that the phase coherence is conserved when the charge carrier enters or leaves the molecule. There is thus no phase loss of the carrier wavefunction at the metal/molecule interface. For simplicity, we use the simplest possible models for the electrode and the molecule that still retain atomistic structure. We employ a one-orbital-per-site tight-binding model for the electrode, which we assume is one-dimensional and semi-infinite. The electron hopping inside the electrode is also assumed to be coherent, with no electron-phonon interactions. The following parameters describe the electrode and its connection to the molecule: $\varepsilon_{L, R}$ is the on-site energy, $\beta_{L, R}$ is the inter-site hopping matrix 
element within the electrode and $v_{L, R}$ is the matrix coupling element from the molecule ends to the left (' $L$ '), right (' $R$ ') electrode.

Now we briefly describe the model used for the molecular wires and the technique that we have developed to calculate the carrier injection into the molecule. We use a version of the $\mathrm{Su}-\mathrm{Schrieffer-Heeger}(\mathrm{SSH})$ model (Heeger et al 1988) in which the $\pi$-electrons are described by a simple tight-binding model and the $\sigma$-bonds as a network of harmonic springs, and in which the lattice motion is fully quantized. The Hamiltonian is

$$
\begin{gathered}
H=t \sum_{l \in \text { left }}\left(d_{l}^{+} d_{l+1}+\text { h.c. }\right)+\sum_{n} \varepsilon_{n} c_{n}^{+} c_{n}+\sum_{n m \lambda} \gamma_{n m \lambda} c_{n}^{+} c_{m}\left(a_{\lambda}+a_{\lambda}^{+}\right) \\
+\sum_{\lambda} \hbar \omega_{\lambda}+\sum_{n} V_{n}\left(c_{n}^{+} d_{0}+\text { h.c. }\right)
\end{gathered}
$$

where $c_{n}^{+}\left(c_{n}\right)$ creates (annihilates) an electron in the $n$th electronic state with energy $\varepsilon_{n}$. These states are taken to be the one-electron eigenstates of the ground state reference system; in the case of conjugated polymers, they correspond to $\pi$-electrons delocalized along the molecular axis. The electrons interact with the vibrational modes $\lambda$ of the molecule $\left(a_{\lambda}^{+}\right.$creates a quantum of energy $\hbar \omega_{\lambda}$ in the mode $\lambda$ ) via the electron-phonon matrix elements $\gamma_{m n \lambda}$. The parameters $\varepsilon_{n}, \omega_{\lambda}$ and $\gamma_{\lambda m n}$ of the model Hamiltonian are obtained from the ground state of the isolated neutral molecule containing $N$ monomers; this neutral molecule is described by the SSH model (Heeger et al 1988). The harmonic approximation is then used to obtain the vibrational eigenmodes $V_{\lambda}$ and frequencies $\omega_{\lambda}$ of the molecule (Chao and Wang 1985).

We work throughout in the coherent transport regime; the carrier injection properties (for the single-metal/molecule interface geometry) are then determined by using a stationary-state inelastic scattering technique. The scattering states $|\Psi(E)\rangle$ for a single incoming (injected) charge carrier are expanded into the eigenstates

$$
\left|n,\left\{n_{\lambda}\right\}\right\rangle=c_{n}^{+} \prod_{\lambda} \frac{\left(a_{\lambda}^{+}\right)^{n_{\lambda}}}{\sqrt{n_{\lambda} !}}|0\rangle
$$

of the non-interacting electron-phonon system, where $n_{\lambda}$ is the occupation number of the vibrational mode $\lambda$. For the vacuum state $|0\rangle$, we take the ground state of the whole system which contains a definite number of electrons in each part (the molecule is initially considered to be neutral). The states $\left|n,\left\{n_{\lambda}\right\}\right\rangle$ correspond to adding a single carrier to this reference state and adding a definite number of vibrational quanta to each mode $\lambda$. The electron added to the system can then be anywhere-in the electrode or in the molecule (where it interacts with the lattice vibrations). This assumption implies that only scattering states in which a single electron (or hole) is added to the ground state of the neutral molecule are considered. We expect this assumption to be valid, even for coherent transport through the whole molecule, because the electron residence time is much shorter than the time interval between two successive electron processes (Ness et al 2001).

This scattering problem is solved (and the transport or charge injection properties of the molecule obtained) by transforming the many-body problem into a single-electron problem with many scattering channels (Bonca and Trugman 1995). Each of the possible scattering process between the single incoming carrier and the vibrational modes is associated with a different channel. We have already shown that the problem is solved once the value of the scattering state $|\Psi(E)\rangle$ inside the molecule is known (Ness and Fisher 1999, Ness et al 2001). Such a state is obtained for an initial energy $E$ by propagation of the source term $|s(E)\rangle$ (representing an incoming carrier from one electrode):

$$
|\Psi(E)\rangle=G(E)|s(E)\rangle .
$$


The propagator or Green function $G(E)$ defined in the molecule subspace is given by

$$
G(E)=\left[E-\hat{H}-\Sigma_{L}(E)-\Sigma_{R}(E)\right]^{-1},
$$

where $\hat{H}$ is the molecular wire Hamiltonian defined previously. By projecting out the basis set associated with the electrodes, one can work solely in the molecular subspace. This projection introduces the so-called self-energies $\Sigma_{L, R}$ in the definition of $G(E)$. This technique is similar to the embedding technique which effectively permits one to reduce the size of a Hamiltonian matrix to the region/subspace of interest. The complex potentials $\Sigma_{L, R}(E)$ arise from this Hamiltonian size reduction (i.e. embedding the spectrum of the molecule into the electrodes continuum of electronic states) and are dependent on the energy $E$.

Once the linear system $|\Psi(E)\rangle=G(E)|s(E)\rangle$ is solved, the scattering state $|\Psi(E)\rangle$ is known and one can also calculate all expectation values, in particular the value $\langle f(c, a)\rangle=$ $\langle\Psi(E)|f(c, a)| \Psi(E)\rangle$ of any correlation function $f$ connecting the electron and phonon degrees of freedom. For example, we can find the atomic displacements induced by the injection of an electron into the molecular wire at a given point. We do this by calculating the expectation value $\delta_{\lambda}^{i}$ of the following correlation function connecting the electron density at a particular site and the displacement of a given normal mode:

$$
f(c, a) \equiv c_{i}^{\dagger} c_{i}\left(a_{\lambda}^{\dagger}+a_{\lambda}\right) \sqrt{\frac{\hbar}{2 M \omega_{\lambda}}},
$$

where $c_{i}^{\dagger}=\sum_{n}\langle n \mid i\rangle c_{n}^{\dagger}$ creates an electron on site $i$. $\delta_{\lambda}^{i}$ gives the displacement of the vibrational mode $\lambda$ induced by the corresponding electron density $c_{i}^{\dagger} c_{i}$ on site $i$. The normal-mode displacements can then be used to obtain the response of the lattice in the molecular chain. The corresponding atomic displacements are calculated as $\delta u_{j}^{i}=\sum_{\lambda} \delta_{\lambda}^{i} V_{\lambda}(j)$.

Finally, for a given initial total energy $E$, the boundary conditions need to be chosen. In this paper, we consider the limit of low temperatures where the initial occupations of the vibrational modes $b \equiv\left\{m_{\lambda}\right\}=0$ correspond to the ground state of the harmonic oscillators. This is a good approximation even at room temperature for all optic modes, which are the modes most strongly coupled to the injected charge. However, when a soliton defect is present in the molecule, the low frequency of the mode associated with the soliton translation restricts us to lower temperatures; the condition $k T \ll \hbar \omega$ then gives $T \ll 200 \mathrm{~K}$. A single charge carrier is injected from the left electrode into the molecule. Within our tight-binding model, the initial energy of the electron (or hole) is given by $\varepsilon_{\text {in }}=\varepsilon_{L}+2 \beta_{L} \cos \left(k_{b}^{L}\right)$, where $k_{b}^{L}$ is the (dimensionless) wavevector of the corresponding incoming wave.

The outgoing charge reflected back to the left has an energy $\varepsilon_{\text {out }}=\varepsilon_{L}+2 \beta_{L} \cos \left(k_{a}^{L}\right)$ where $a \equiv\left\{n_{\lambda}\right\}$ is the occupation distribution of the modes after the scattering. Since in our model there is no overall dissipation (the vibrational modes $\lambda$ are not coupled to any further dissipative bath, either in the molecule itself or in the electrode), the system conserves its total energy $E$. The electron and vibrational mode energies, before and after scattering, are thus related by

$$
E=\varepsilon_{\text {in }}+\sum_{\lambda} m_{\lambda} \hbar \omega_{\lambda}=\varepsilon_{\text {out }}+\sum_{\lambda} n_{\lambda} \hbar \omega_{\lambda}
$$

In the limit of low temperatures, where the vibrational modes are initially in the ground state, the electronic injection energy $\varepsilon_{\text {in }}$ equals the total energy $\left(\varepsilon_{\text {in }}=E=\varepsilon_{\text {out }}+\sum_{\lambda} n_{\lambda}^{\hbar} \omega_{\lambda}\right)$.

\subsection{Charge injection: results}

In this section, we concentrate on results for the coherent process of carrier injection from an electrode into the molecule. In the context of the overall process of transport through a 


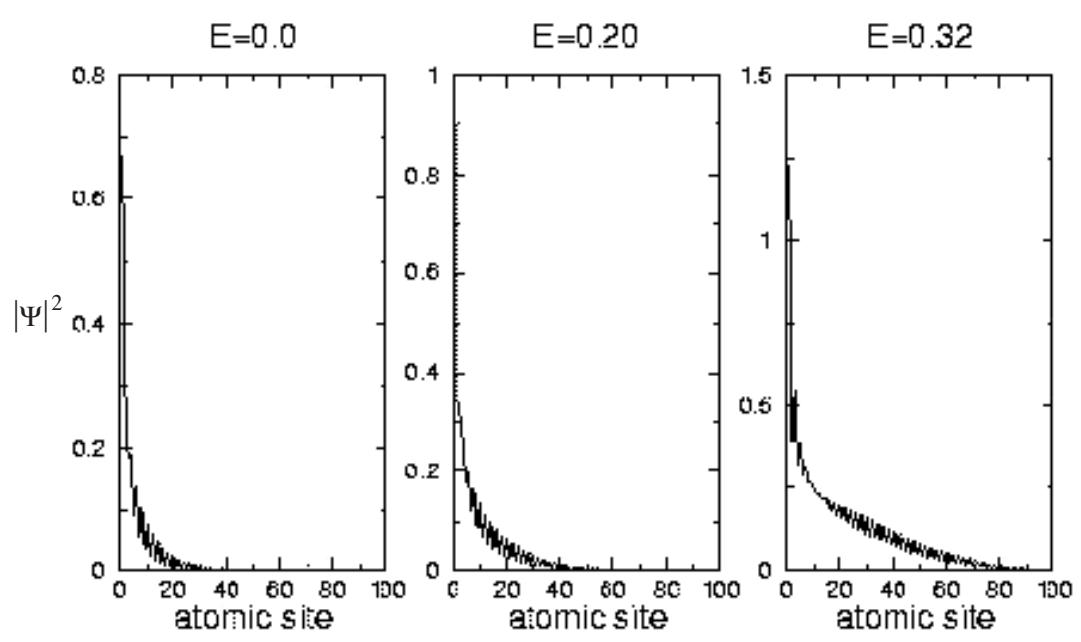

Figure 2. Total electron density $|\langle j \mid \Psi(E)\rangle|^{2}$ on atomic site $j$ calculated for different injection energies $E$ inside the HOMO-LUMO gap of the molecule. The molecular wire contains $N=100$ monomers. Note that (for any molecule length) the energy reference $E=0$ is chosen to be exactly at mid-gap between the HOMO and LUMO levels of the corresponding molecule. The electron density decays exponentially from the metal/molecule interface $(j=0)$ into the molecule itself; this is similar to the decay of electron density inside a tunnelling barrier.

molecule or a molecular film, this may be considered as the first of a sequence of incoherent transport steps. It might be followed by any of a number of different steps inside the molecule (for example, electron/hole recombination, rearrangement/relaxation of the structure of the molecule, injection of extra charge carriers); the important assumptions are that the coherence of different portions of the wavefunction has been lost before any of these subsequent processes occur and that the transport proceeds by a sequence of different and effectively uncorrelated events.

The injection process itself, however, we consider to be a single, coherent event. It is complicated by the presence of an associated structural relaxation, due to the interaction between the injected carrier and the atomic displacements of the molecule. The electronphonon coupling producing this relaxation is precisely what was described in the previous section. The molecule is then connected to a single metallic electrode (the left electrode) by one end. There is no net current passing inside the molecule; however, an electronic wavefunction can penetrate from the metal into the molecular wire. Owing to the electronphonon coupling, the amplitude of the incoming electronic wavefunction is distributed into the different elastic and inelastic channels.

The energy of the electron wavefunction depends on the position of the Fermi level of the metallic electrode relative to the molecular orbitals of the wire. Such energy level alignment is, in principle, determined by the exact self-consistent solution of the problem including charge transfer between the metal and the molecule. However, for the model used here, we consider the energy $E$ of the incoming electron as a variable, in order to explore the different possible physical conditions. We are therefore able to obtain qualitative results, which should be valid for a range of different electrode materials (corresponding to a range of different positions for the Fermi energy within the HOMO-LUMO gap of the molecule).

For an initial energy $E$ at mid-gap, the total injected electron density $|\langle j \Psi(E)\rangle|^{2}$ at site $j$ is shown in figure 2 . The overall shape of the electron density is characteristic of an exponentially 

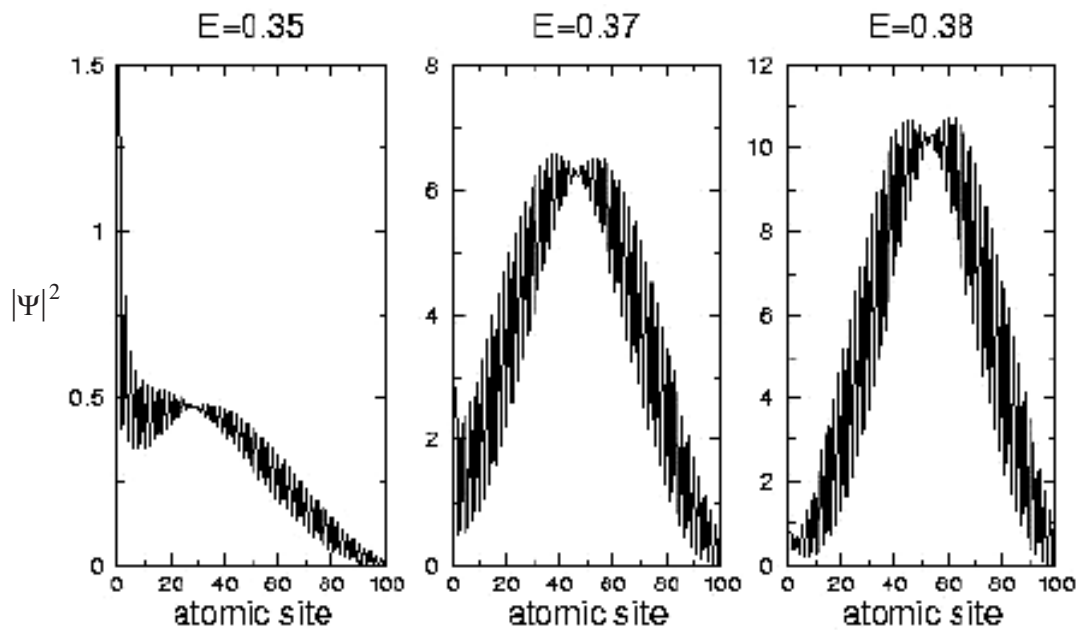

Figure 3. Electron density injected inside the molecule (length $N=100$ ) for energies $E$ approaching the band edge. At the polaron resonance (energy around $0.38 \mathrm{eV}$ ), the electron is mostly localized at the middle of the molecule. The electron density is then more symmetric with respect to the molecule's centre, in contrast to the 'purely tunnelling' case (figure 2).

decaying wavefunction inside a tunnelling barrier. The small-wavelength oscillation in the electron density comes from the discrete nature of the molecular wire and from the intrinsic shape of the molecular one-electron states $|n\rangle$ near the HOMO-LUMO gap that are used to construct $|\Psi\rangle$. For energies around mid-gap (for example $0 \leqslant E \leqslant 0.20 \mathrm{eV}$ in the case of a $N=100$ chain length), the decay is approximately exponential, with a decay rate $k(E)$ that roughly follows the usual law $k(E) \propto \sqrt{\left(V_{0}-E\right)}$ : the electron density decays less rapidly as the energy $E$ approaches the 'barrier height' $V_{0}$. However, owing to the electron-phonon coupling, this behaviour occurs only for injection energies located within a limited energy range deep inside the HOMO-LUMO gap of the molecule. For higher energies, the electron density behaves somewhat differently, as shown in figure 3. Furthermore, one might expect the effective barrier height $V_{0}$ felt by the injected electron to be given by the first eigenvalue just above the gap, i.e. the energy of the LUMO state (for example, for $N=100$, our model gives $E_{\mathrm{LUMO}}=0.48 \mathrm{eV}$ ). Such a picture is only correct in the absence of electron-phonon interactions. In the presence of electron-phonon coupling, the apparent 'barrier height' is lower. This is because the injected electron (even for charge injection below the gap) induces distortions in the molecular lattice. We have already identified such distortions as being associated with the formation of a virtual polaron in the case of transport through the molecular wire (Ness et al (2001), Ness and Fisher (1999); see also next section). The polaron formation is also associated with a relaxation energy of the system. In the simpler case of one electron coupled to a single phonon mode (frequency $\omega$ and electron-phonon coupling constant $\gamma$ ), the corresponding relaxation energy is $\gamma^{2} / \hbar \omega$ and shifts the eigenvalues accordingly. In our model, where the electron is coupled to several phonon modes via the different coupling matrix elements $\gamma_{\lambda m n}$, the expression for the relaxation energy is more complex but the same physics remains: one observes an effective lowering of the 'barrier height'.

For injection energies $E$ increasing towards the valence band edge, the electron density progressively loses its purely tunnelling character (figure 2). One observes that the electron density takes the shape of a resonance mostly located at the centre of the molecule, especially for injection energies at (or around) the polaron resonance observed in the curves for electron 


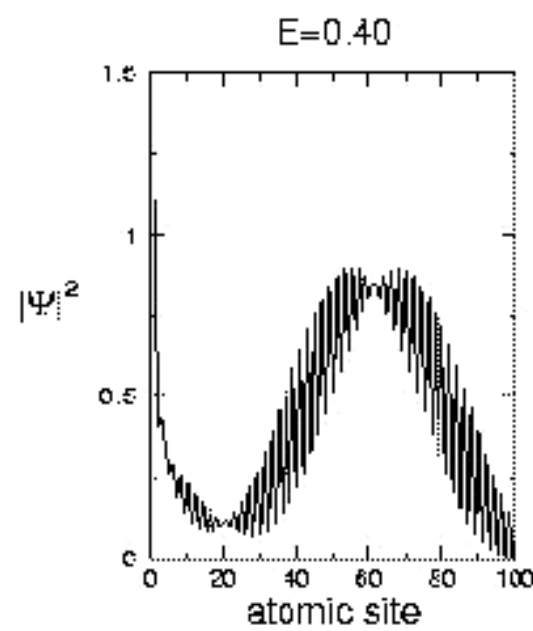

Figure 4. Electron density injected inside the molecule (length $N=100$ ) for an energy $E$ above the polaron resonance energy but still below $E_{\text {LUMO }}$. The density has an exponentially decaying behaviour at the metal/molecule interface and exhibits a peak in the middle of the molecule.

transmission (Ness et al 2001). For a chain of length $N=100$, this happens for injection energies around $0.37 \mathrm{eV}$.

Above the polaron resonance but below the LUMO energy, the electron density displays both behaviours. The density decays exponentially from the metal/molecule interface into the molecule as shown in figure 4. The curve also has a peak of density in the middle of the molecule, indicating that part of the electron density is located around the centre of the molecule.

The variations of the electron wavefunction versus the different energies $E$ are also reflected in the mean position of the injected electron. From the norm-normalized scattering wavefunction, we calculate the mean position $\left\langle x_{i}\right\rangle=\left\langle\Psi(E)\left|x_{i}\right| \Psi(E)\right\rangle$ of the electron inside the molecule. Figure 5 shows that for injection energy $E$ deep inside the HOMO-LUMO gap, the electron penetration is weak (most of the electron density is located at the end of the molecule connected to the metallic electrode). As seen before, for increasing $E$-values, the electron penetration at first increases slowly. Then, for injection energies around the polaron resonance, the electron position increases much more rapidly. At the resonance the electron is localized to some extent at the middle of the molecule.

\section{Carrier processes within the polymer chain}

Once a carrier has entered a chain, what are the essential features of its motion? Whilst this problem has been studied far more than carrier injection, unfortunately most of the work (e.g. Heeger et al 1988) has been in the context of isolated long chains. This is very much an idealization. We shall use the informal word 'chain' to describe a polymer molecule, recognizing that the chain may be relatively short and may be bent, or branched, or crosslinked in some way. In a typical semiconducting polymer, the molecules are intertwined. Most of the individual conjugated segments have about 10 monomer units or less; there is also a statistical distribution of overall molecular lengths, although this is generally made as uniform as is practicable. The molecule can be designed to be soluble in particular solvents, or to have additional functionality. 


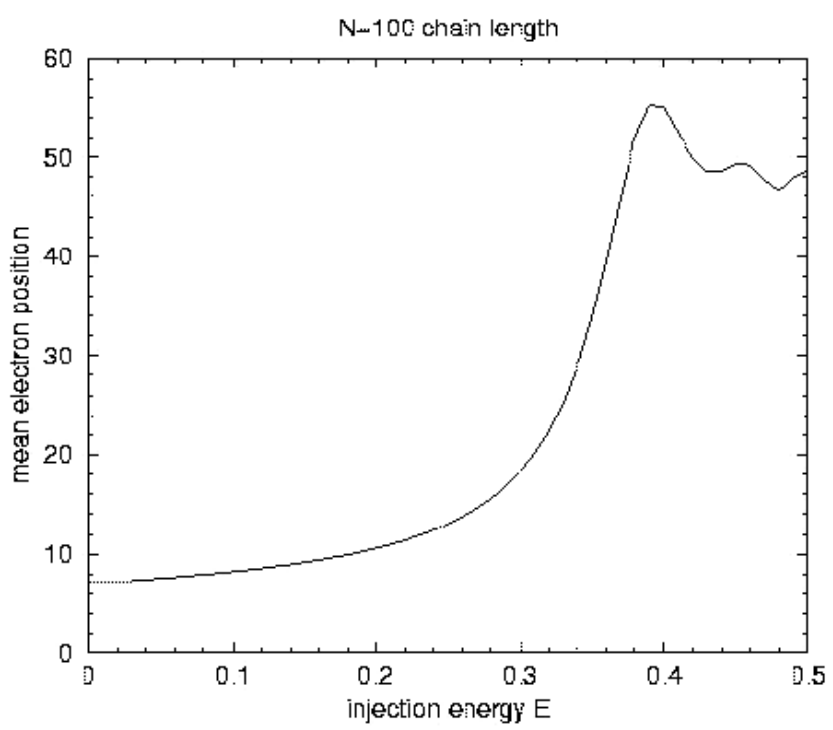

Figure 5. The expectation value of the position $\left\langle x_{i}(E)\right\rangle$ of the injected electron in the molecule versus the injection energy $E$. The molecule is made of $N=100$ monomers. For injection at mid-gap, the electron is found close to the end of the molecule connected to the electrode. At the polaron resonance (around $0.38 \mathrm{eV}$ ), the electron is located at the middle of the molecule.

In describing the carrier processes, we shall need both the mesoscopic 'spaghetti' structures, to be discussed separately in section 4 , and a range of predictions relating to either a single chain or to two chains. The properties needed are of four main types. First, we shall need fully relaxed energies for chains, with dependence on chain lengths, curvatures and similar features. The relaxed energy is appropriate when the inter-chain hopping is slow compared with relaxation times, as seems to be generally the case. The same calculations should provide charge densities accurate enough for discrete space-charge calculations. Secondly, we shall need relaxation energies for both injection and recombination processes which, with phonon energies, form the basis for calculations of optical line shapes and non-radiative transitions. Thirdly, intra-chain carrier mobilities are needed. These can be obtained from computer experiments in which evolution is observed in an applied field. Fourthly, we shall need to have a means to estimate inter-chain jump probabilities. This problem resembles that of the injection process of section 2. The longer-range part of the wavefunction will be necessary for quantitative calculations, and is not accurately represented in simpler methods.

Methods are available for electronic structure at several levels, usually with some sort of simple atomic orbital basis. The model of section 2 follows the SSH approach, using empirical parameters. This approach yields insight, and has been very widely used, but some behaviour may be hard to analyse empirically. The simpler, empirical, molecular orbital methods, like extended Hückel theory, can also be illuminating, but the lack of self-consistency is a weakness, and usually the total energy is taken from a sum of one-electron eigenvalues, which introduces errors. More useful are the simple self-consistent methods, again semi-empirical, like the CNDO technique. As with all methods mentioned so far, there will be errors associated with the limited basis, notably in phonon energies; excitation energies can be a problem for all methods, including some so-called a priori approaches. The advantages of methods like the CNDO technique is that they are systematic, any problems like convergence errors are usually easy to spot and there is a huge amount of experience in the chemical literature. The CNDO 

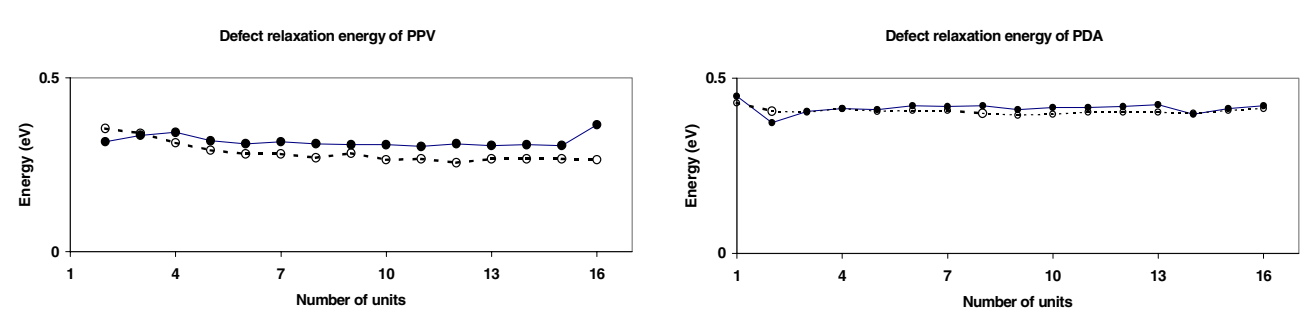

Figure 6. Relaxation energies of positive (open symbols) and negative (filled symbols) polarons as a function of chain length for PPV (left) and PDA (right). Note that the relaxation energies are approximately independent of length, even for relatively short chains.

method has been embodied into molecular dynamics (CHEMOS; see Wallace et al 1991a), with forces calculated at each time step without numerical differentiation. This class of code is very good for getting a feel for possible behaviour. It also provides a preliminary to more sophisticated work. The state-of-the-art methods, such as local density methods or HartreeFock methods, have not been used so much for dynamics. They share with simpler methods some of the problems in treating excited states, and are sometimes used empirically (e.g., in choosing whether or not to use gradient corrections).

The calculations described in this section use CHEMOS (Wallace et al 1991a, 1991b), i.e., the CNDO level with self-consistent molecular dynamics. This gives us consistent guidance as to some quite sophisticated effects, e.g., at high electric fields $\boldsymbol{E}$, and simple estimates of the effects of chain length, curvature and the like. We recognize that the electronic energies are overestimated (to a degree that is semi-systematic) and there is corresponding overestimation of vibrational frequencies.

\subsection{Static properties: energetics and distortions}

Using the semi-empirical approach, one can calculate the full range of static properties for a chain in its neutral or charged states. The energy information for the relaxed state includes the ionization potential IP (the energy needed to remove an electron or add a hole), the electron affinity EA (the energy gained on adding an electron) and the chemical potential CP, $\mu=(\mathrm{IP}+\mathrm{EA}) / 2$. The excitation energy at constant geometry (the Franck-Condon transition) and the subsequent relaxation energy $E_{R}$ are available. We may study singlet or triplet states. The nature of the relaxation is important, notably the dimerization. If $u_{n, n-1}$ is the bond length between the $n$th and $n-1$ th atoms, then the dimerization is $\delta=(-1)^{n}\left(u_{n, n-1}-u_{n, n+1}\right)$. The dimerization is especially useful in following carrier dynamics (section 3.2).

Following the injection of a single charge (electron or hole) into a PDA or a PPV chain, a geometric relaxation appears localized over about four monomer units owing to the strong charge-lattice coupling (Ramos et al 2001, 2002, Almeida et al 2001). Our CHEMOS calculations predict a greater distortion than do other methods (Cornil et al 2001). The relaxation energy of positive and negative defects is almost independent of the oligomer size (figure 6). In describing charge transport along the polymer strands, it is useful to consider how the charge induces a structural defect in the chain. Our results suggest that injected charge localizes at the distortion site, and is mainly stored on the carbon atoms (55\% in PPV and $72 \%$ in PDA; see Ramos et al 2002), showing an alternating pattern (Ramos et al 2001, 2002, Almeida et al 2001).

We can predict the IP, EA and chemical potential $\mu$ as a function of the number of monomer units $N$ in the chain. For some previous calculations (e.g. those for trans-polyacetylene; see 

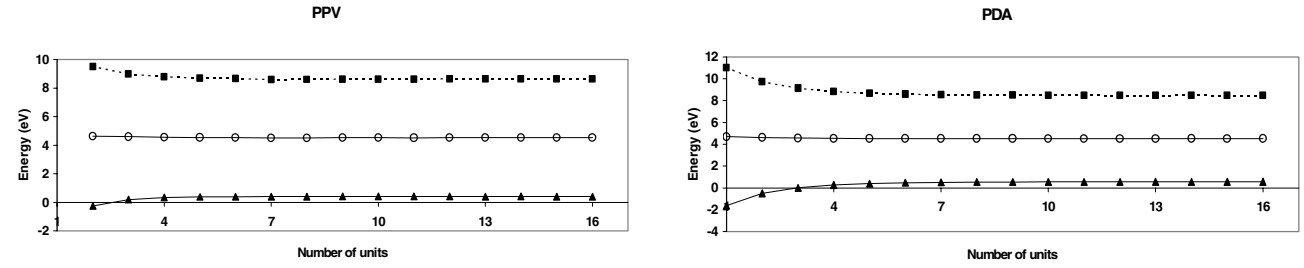

Figure 7. Ionization energy (filled triangles), electron affinity (filled squares) and chemical potential (open circles) as a function of chain length for PPV (left) and PDA (right).

Ramos et al 1994) the predicted IPs agree well the experimental data. In many other cases, including our present work on poly(phenylene vinylene) (PPV) and polydiacetylene (PDA), there are substantial but systematic overestimates of the magnitudes of binding energies. The results are shown in figure 7 for PDA and PPV. The long-chain limit is reached for relatively short chains, indeed faster than one would expect for a fully delocalized electron or hole in a one-dimensional box $\left(E \propto l / N^{2}\right)$. This reflects the fact that the lattice distortion self-traps the carriers. The effects of chain length and curvature (Ramos et al 1994) lead to chain-to-chain variations from a reasonable statistical sample of around $40 \mathrm{meV}$. This variability underlies the distributions of electronic energies discussed later (see section 5). The spread of these energies, the thermal energy $k T$ (about $0.025 \mathrm{eV}$ at room temperature) and the electrostatic energy change on moving a carrier through the length of a chain are all very similar (10 meV for $1 \mathrm{~nm}$ in a field of $10^{5} \mathrm{~V} \mathrm{~cm}^{-1}$ ). Further site-to-site variations in energy can result from proximity to media with different dielectric constants (electrodes or inclusions) and space charges.

Excitation and de-excitation (radiative and non-radiative recombination) are key processes. The relaxations, including changes in dimerization, make a significant contribution to the energetics. This is important for two reasons. First, they relate to the optical spectrum of a single chain in a way which is often described by Huang-Rhys theory (Stoneham 1975). However, we note that a conjugated polymer is not a system to which Huang-Rhys theory strictly applies (because there are many electronic states, and many modes with different frequencies, involved), so Huang-Rhys factors from observables (zero-phonon-line fraction, Stokes shift, linewidth) may not agree. Second, the relaxations affect the variation of energies from one chain to another. Singlet excitons can be formed by optical excitation; singlet or triplet excitons can be formed by the combining of electrons and holes in the polymer. Ramos et al (1994) show the evolution of the dimerization as an electron and a hole evolve to form an exciton. Since there are many possible electron and hole states, one should not assume that the same states are formed under different forms of excitation, e.g., using plasmons instead of photons (see, e.g., Alvarado et al 2001).

In our models, excitons are spatially limited to one excited molecule. Inter-molecular recombination is possible and could be treated, but we have not attempted this because we have reservations about the accuracy possible within a simple tight-binding basis. There is local geometry relaxation in the central region of the polymer chain associated with both singlet and triplet excitons in both PDA and PPV. In both polymers (PDA and PPV), the width of the distortion in a singlet exciton is predicted to be about one lattice unit greater than that of a triplet exciton. The extent of singlet exciton distortion is greater in PDA (six units, compared to four units in PPV).

Exciton formation leads to charge rearrangements among the polymer atoms. These rearrangements are greatest for the carbon atoms, as might be expected, since the states either 

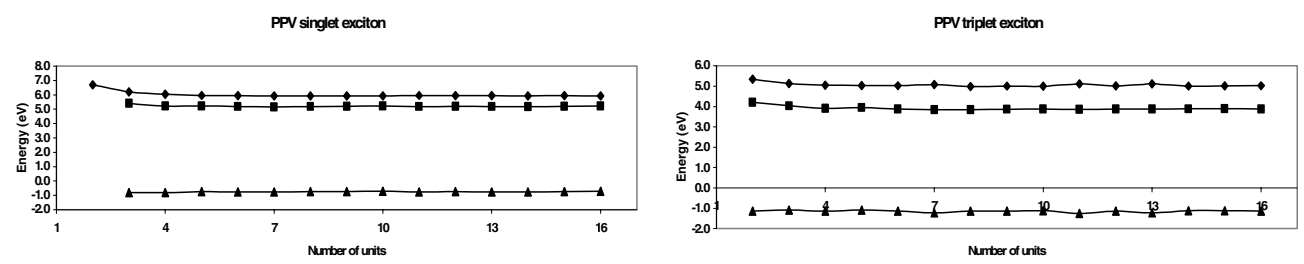

PDA singlet exciton
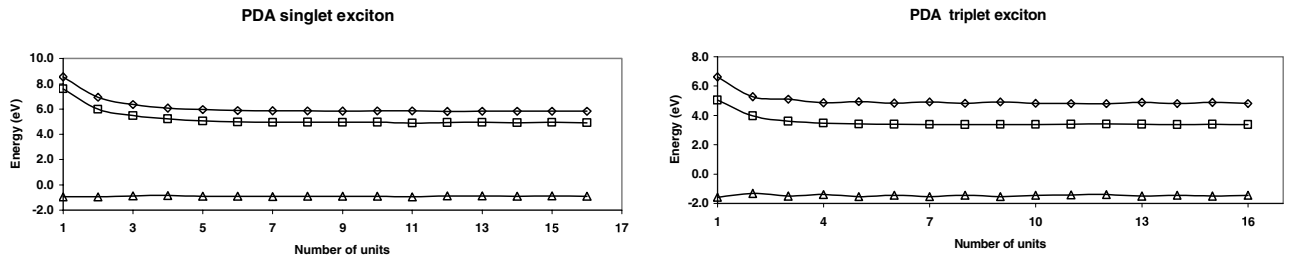

Figure 8. Excitation energy (diamonds), exciton energy (squares) and relaxation energy (triangles) of singlet (left) and triplet (right) excitons as a function of chain length for PPV (top) and PDA (bottom).

Table 1. Field-dependent mobilities extracted from the experiments of Tessler et al (1998) (middle two columns) and as calculated (this work, third column).

\begin{tabular}{llll}
\hline & Tessler et al $($ low $E)$ & Tessler et al (high E) & Present work \\
\hline Carrier density $\left(10^{17} \mathrm{~cm}^{3}\right)$ & 0.1 & 1.6 & 44 \\
Current density $\left(\mathrm{A} \mathrm{cm}^{-2}\right)$ & 101 & 600 & 40 \\
Mobility $\left(\mathrm{cm}^{2} \mathrm{~V}^{-1} \mathrm{~s}^{-1}\right)$ ohmic; & $0.001-0.002$ (ohmic); & 0.075 (ohmic); & $2 \times 10^{-5}$ \\
space-charge-limited current & 0.08 (SCLC) & 0.04 (SCLC) & 2.5 \\
Electric field $\left(10^{6} \mathrm{~V} \mathrm{~cm}^{-1}\right)$ & 1.2 & 3 & \\
\hline
\end{tabular}

side of the HOMO-LUMO gap are predominantly carbon $\pi$-states. As might also be expected from the trapping of the excitons, this charge rearrangement of carbon atoms is localized near the distortion site. For triplet excitons we find a charge alternation on both carbon and hydrogen atoms, whereas the formation of a singlet exciton leads to charge storage of opposite sign across the defect on both carbon and hydrogen atoms. Charge of opposite sign is also stored on hydrogen and carbon atoms of the same unit.

For PDA and PPV, both singlet and triplet exciton energies decrease with chain size but (like the IP and EA results) converge to a nearly constant value, the effect being more pronounced for PDA. These trends agree with those obtained from the optical absorption spectra of a diacetylene crystal after photo-initiation and thermal reactions (Sixl 1984). The relaxation energies of both triplet and singlet excitons for PDA and PPV are roughly constant with chain length (figure 8). As noted previously, the calculated trends in IP and EA with chain length are qualitatively correct. Similarly, even though the absolute values of calculated singlet and triplet exciton energies may not be correct, we expect reliable predictions of trends on varying the chain length for PDA and PPV. The calculated singlet exciton energies of trimer, tetramer, pentamer and hexamer molecules are $3.2 \mathrm{eV}$ higher than corresponding experimental optical absorption energies (Sixl 1984). If we were to assume that the singlet exciton energy for PPV is also $3.2 \mathrm{eV}$ higher than the experimental ones, then our results would suggest that PPV produces a yellow-green luminescence in agreement with photoluminescence spectra (Friend et al 1999). 

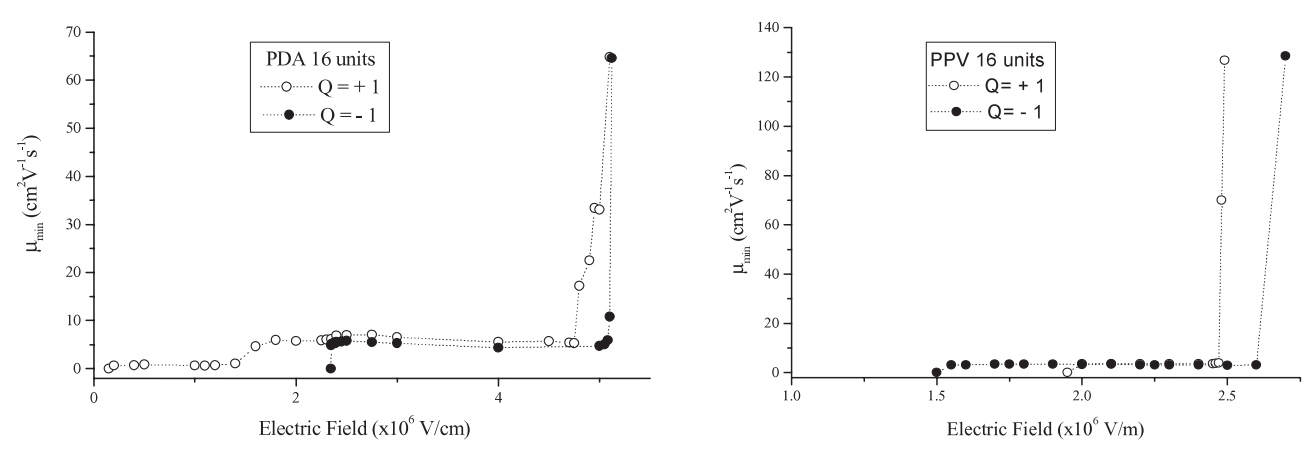

Figure 9. Calculated intra-molecular mobility as a function of electric field for PDA (left) and PPV (right), showing results for holes (empty circles) and electrons (filled circles). In both cases the molecule was 16 repeat units long.

Table 2. Electric field ranges for low and high intra-molecular charge mobility $\left(\mathrm{cm}^{2} \mathrm{~V}^{-1} \mathrm{~s}^{-1}\right)$ in PPV and PDA chains with 16 repeated units.

\begin{tabular}{lll}
\hline Polymer & Net Charge & Electric field threshold $\left(10^{6} \mathrm{~V} \mathrm{~cm}^{-1}\right)$ \\
\hline PDA & -1 & $2.35[5]$ \\
& +1 & 1.60 \\
PPV & -1 & $1.55[5]$ \\
& +1 & 2.0 \\
\hline
\end{tabular}

In assessing electron-hole recombination, there are several important factors. One is the symmetry of the exciton wavefunctions: luminescence is only strong when the excited state symmetry allows a dipole coupling to the ground state. The most common case is one where the system possesses inversion symmetry and the ground state has the symmetry of the identity representation, $A_{g}$. The excited state must then have $B_{u}$ symmetry for the transition to be dipole allowed. Exciton wavefunction symmetry is important for both radiative and non-radiative transitions (see e.g. Cornil et al 2001).

\subsection{Carrier dynamics: intra-chain mobility}

Experimentally, it is found that carriers can have respectable intra-chain mobilities, but these are still low relative to those of most conventional semiconductors. Measurements by Hoofman et al (1998) for a soluble derivative of PPV (poly(phenylene vinylene)) give intra-chain mobilities of $\mu_{e}=0.15 \mathrm{~cm}^{2} \mathrm{~V}^{-1} \mathrm{~s}^{-1}$ and $\mu_{h}=0.06 \mathrm{~cm}^{2} \mathrm{~V}^{-1} \mathrm{~s}^{-1}$. Bulk values, which involve inter-chain hops, are usually significantly less, and depend on various aspects of preparation. However, Tessler et al (1998) find relatively high hole mobilities in PPV. They discuss two analyses: mobilities assuming ohmic behaviour and those assuming space-chargelimited currents. For ohmic behaviour, the mobilities rise with applied field, but do not exceed $0.01 \mathrm{~cm}^{2} \mathrm{~V}^{-1} \mathrm{~s}^{-1}$. For space-charge-limited currents, the mobility falls with applied field from $0.08 \mathrm{~cm}^{2} \mathrm{~V}^{-1} \mathrm{~s}^{-1}$ to around $0.04 \mathrm{~cm}^{2} \mathrm{~V}^{-1} \mathrm{~s}^{-1}$. There is also a strong dependence of mobility on the total concentration of injected carriers. Values quoted in the literature include $10^{-6} \mathrm{~cm}^{2} \mathrm{~V}^{-1} \mathrm{~s}^{-1}$ (Jarrett et al 1995). In other systems, values given by Alvarado et al (2001) suggest mobilities of $10^{-3} \mathrm{~cm}^{2} \mathrm{~V}^{-1} \mathrm{~s}^{-1}$ for the hole injection layer of CuPc (copper phthalocyanine) and about $10^{-4} \mathrm{~cm}^{2} \mathrm{~V}^{-1} \mathrm{~s}^{-1}$ for $\mathrm{Alq}_{3}$ (aluminium chelate complex), the electron transport and emitting layer. 
Table 3. Electric field ranges for low and high intra-molecular charge mobility $\left(\mathrm{cm}^{2} \mathrm{~V}^{-1} \mathrm{~s}^{-1}\right)$ in PPV and PDA chains with 16 repeated units.

\begin{tabular}{llllll}
\hline & \multicolumn{3}{c}{ Electric field $\left(10^{6} \mathrm{~V} \mathrm{~cm}^{-1}\right)$} \\
\cline { 2 - 3 } \cline { 5 - 6 } Polymer & Low mobility & High mobility & & Low mobility & High mobility \\
\cline { 2 - 5 } \cline { 5 - 6 } PPV & {$[1.55-2.60]$} & {$[2.70-11.00]$} & & {$[2.00-2.47]$} & {$[2.49-11.00]$} \\
PDA & {$[2.35-5.08]$} & {$[5.12-14.70]$} & & {$[1.60-4.70]$} & {$[5.10-14.90]$} \\
\hline
\end{tabular}

Theoretically, we can estimate mobilities using the CHEMOS code. To do this, we use the definition of mobility as velocity per unit applied field (Wallace et al 1991b). In essence, we measure the velocity in self-consistent molecular dynamics as the velocity of either the charge density or of some clear feature of the dimerization $\delta$ (e.g., $\delta=0)$ following the imposition of an electric field. Our present results are shown in table 1.

There is a clear field dependence of the mobility, with three main regimes. First, when the field is lower than the threshold, the injected charge does not move from the central region. This seems to imply that the electronic polarizability should show a threshold behaviour when there are carriers but blocking contacts. Second, for a moderate electric field above the threshold, the charge mobility is low and both charge and distortion patterns move slowly. Third, in the high-field regime the intra-molecular charge mobility seems to be much higher. However, it may be simply that the assumption of a mobile carrier and its associated deformation field is now inadequate, and that in fact the coupling between injected charge and induced defect is broken. In our simulations, the charge is already found at the chain end on the first time step, whereas the distortion pattern vanishes while a new structural defect arises at the new charge site. Both distortion patterns oscillate with time. The transition between the moderate- and high-field regimes is sharp (see figure 9). The method used in these simulations becomes unreliable, and indeed self-limited, since when the field goes beyond $11 \times 10^{6} \mathrm{~V} \mathrm{~cm}-1$ for PPV and $15 \times 10^{6} \mathrm{~V} \mathrm{~cm}^{-1}$ for PDA, the electronic self-consistency procedure fails to converge.

Since mobilities are inferred, rather than measured directly, any comparison of theory and experiment needs to make assumptions about the carrier density. One can deduce results from the extreme cases shown in figure 4 of Tessler et al (1998). Comparing our theoretical results (tables 2 and 3) with those inferred from these experiments, we see that our theoretical figures suggest too large a carrier density. This is partly because the mobility used is low (but not unreasonably so). It is possible, however, that the carrier density will also make a difference to the transport through the importance of space-charge discreteness in producing 'Coulomb glass behaviour (Stoneham and Ramos 2001; see also below). Tessler et al (1998) talk of an exciton density at high currents of $10^{14} \mathrm{~cm}^{-3}$, which is still low compared with the values estimated by Tessler et al (1996) to be needed for lasing in microcavities with metallic mirrors. In contrast, Harrison et al (1993) used the optical cross-section per injected charge and the reflectivity to estimate carrier numbers. The highest values reported by Tessler et al (1998) are $1.6 \times 10^{17} \mathrm{~cm}^{-3}$. Other intra-chain mobilities which are quite large are given in Hoofman et al (1998), who give $\mu_{e}=0.15 \mathrm{~cm}^{2} \mathrm{~V}^{-1} \mathrm{~s}^{-1}$ and $\mu_{h}=0.06 \mathrm{~cm}^{2} \mathrm{~V}^{-1} \mathrm{~s}^{-1}$.

We have also examined the effect of the applied electric field on PPV triplet exciton transport along the chain. No exciton transport is seen for fields below $8.7 \times 10^{6} \mathrm{~V} \mathrm{~cm}^{-1}$. Beyond this value, and up to $9.9 \times 10^{6} \mathrm{~V} \mathrm{~cm}^{-1}$, the self-consistency loop fails to converge. However, for the applied fields of $10 \times 10^{6} \mathrm{~V} \mathrm{~cm}^{-1}$, the triplet exciton decays into a static isolated polaron pair of different signs localized at opposite chain ends. 


\subsection{Radiative and non-radiative transitions}

Near the electrodes of a LED, non-radiative recombination may involve the conduction electrons of the electrode (see, e.g., Becker Burns et al 1997). Within the bulk of the organic semiconductor, the important competition in electron-hole recombination is between the emission of light and the creation of heat. In principle, an important factor in both processes is the line-shape function $G(E)$ (see, e.g., Stoneham 1975, 1981, Itoh and Stoneham 2001), which measures how the system (here the chain plus its environment) responds to the almost instantaneous forces on change of electronic state. In essence, $G(E)$ relates to the ease with which energy $E$ can be taken up by the vibrational system. For transitions between the same electronic states, both the radiative and non-radiative transitions involve the same line-shape function, although the energy $E$ involved is different, mainly because of the photon energy for the radiative case. Symmetry is involved in both transitions: for radiative transitions, dipole-allowed transitions are by far the most important; for non-radiative transitions, it is the phonon symmetries which matter. Radiative transitions become increasingly important at large transition energies, because of the increased phase space available, whereas non-radiative transitions fall off, since more and more quanta of vibrational excitation are needed. This might be the reason that shorter chains of PDA radiate, whereas longer chains (with lower transition energies) do not do so (Stoneham 1991), although such situations will be rare.

Suppose that phonons of a single energy $\hbar \omega$ dominate in $G(E)$. Two critical parameters are the energy $E$ to be taken up as vibrational energy, in units of the phonon energy (usually $p=E / \hbar \omega)$, and the relaxation energy in units of the phonon energy, $S=\frac{E_{R}}{\hbar \omega}$ with the value $S_{0}$ at zero temperature (the Huang-Rhys factor, in one of its definitions). Since Huang-Rhys theory does not strictly apply to these systems, the Huang-Rhys factors obtained from the zero-phonon-line fraction, the Stokes shift and the linewidth may not agree, but the ideas can be used as a guide. There are also selection rules, both those based on spin (singlet versus triplet) and those relating to orbital symmetry. Broadly speaking (see, e.g., Stoneham 1975), one should distinguish between the promoting modes, which enable a non-radiative transition to occur, and the accepting modes, to which energy is transferred. The phonon energy $\hbar \omega$ entering these expressions above is that for accepting modes.

A careful analysis of a number of conducting polymer systems (Wilson et al 2001b) enables one to conclude that $p \gg S_{0}$, with values of $S_{0}$ of order unity. The analysis is based on the energy gap rule, which assumes that, for a range of similar systems, an $n$-phonon process is a factor $\gamma$ less probable than an $(n-1)$ phonon process. The rule assumes that either phonons of a single frequency (usually the largest available) dominate, or that the proportions of the various frequencies are the same for different values of $n$. It is not clear from the data of Wilson et al (2001a, 2001b) whether there is a promoting mode as well as the accepting mode, nor is it certain that the accepting modes are the same for radiative and non-radiative transitions. Certainly, there are vibrations of several different energies in the observed optical spectrum, and these correspond to smaller energies than one would estimate from direct application of the energy gap analysis (Wilson et al 2001a, 2001b) and the observation that it is hard to understand the energy gap rule unless $\gamma$ is in a range of order 1-2 (see, e.g., Stoneham 1981). Using the observed slopes, this range of $\gamma$ implies, for the non-radiative transitions, polymer phonon energy from 0.17 to $0.34 \mathrm{eV}$ and monomer phonon energies from 0.26 to $0.52 \mathrm{eV}$. The luminescence spectra suggest accepting mode energies in the $0.2-0.25 \mathrm{eV}$ range; somewhat larger values are needed for non-radiative transitions of the corresponding monomers.

In this regime, $p \gg S_{0}$, larger $S_{0}$ will lead to larger non-radiative rates (see, e.g., Stoneham 1981, Itoh and Stoneham 2001). At $T=0$, the line-shape function (unnormalized) has the form $\exp \left[p-S_{0}\right]\left(S_{0} / p\right)^{p}$. So what trends with the number of polymer units, $N$, are expected, 
given our calculations of $E_{R}$ ? Both simple arguments and direct calculation show that the relaxation energies $E_{R}$ for PPV and PDA are essentially independent of $N$ beyond some small value $N_{0}$. For the singlet and triplet excitons, the relaxation energy does not vary to the accuracy of the calculation; for PPV, the variations are below $0.1 \mathrm{eV}$ for $N>3$; the same is true for PDA for $N>2$. So $N_{0}$ is very small and (if the phonons have no greater dependence on $N$ ) the key components of $S_{0}$ should be roughly independent of chain length.

Wohlgenannt et al (2002) show that longer chains of a number of oligothiophenes contribute more to optical light emission (contrary to the previous results for PDA). Tada and Onoda (2002) have shown that photoirradiation of polymer LEDs in air using an incandescent lamp $(150 \mathrm{~W})$ causes the electroluminescence to fall rapidly, becoming undetectable in a few minutes. They deduce that photoinduced defects quench luminescence in poly(3alkylthiophene) (PAT), rather than degrading carrier transport through scission of the main chain. There is little effect on the optical absorption, suggesting that most of the main polymer chains survived photodegradation. This seems to indicate either a long exciton diffusion length or strong inter-chain interaction.

\section{Inter-chain recombination}

As noted, accurate calculations of inter-chain transitions need a better basis set than is available in our present codes. For the purposes of mesoscopic calculations, however, one can accept the empirical observation that inter-chain motion is much slower than carrier motion or relaxation within a single chain. We then need to understand the factors which influence the relative rates of transitions. There are three main factors. The first is relative energy. If $\varepsilon$ is the difference in relaxed energies between initial and final states, we know from the principle of detailed balance that there will be a term $\exp (\varepsilon / 2 k T)$ favouring hops to the lower-energy state and a term $\exp (-\varepsilon / 2 k T)$ for the reverse process. There is also a small change in effective activation energy, as in Marcus theory or any of the many similar theories. The differences in energy come from differences in the chains, from applied fields, image fields, or from fields due to space-charge interactions. The fields will influence the most likely directions of jumps. Obviously, an applied field will drive electrons from cathode to anode, but the (discrete) spacecharge field can cause transient trapping, even in the absence of other energy differences. For the systems of interest here, values of $\varepsilon$ can easily be in the 1-10 range.

The second factor concerns matrix elements and the presence of any symmetry restrictions Cornil et al (2001), Johansson and Stafstrom (2001); again, applied or space-charge fields can be important. The third factor is distance. The tunnelling matrix element will be determined by the longer-range parts of the wavefunction, rather than the shorter-range terms which dominate in the energy. The longest-range component has an exponent which is proportional to the square root of the binding energy (see Stoneham (1975), p 131, or Newton (1960)).

Investigation of the implications of inter-molecular couplings for overall device performance is only just beginning. For example, Magoga and Joachim (1999) describe the effect of (fully coherent) inter-chain processes on the overall transport through molecular wires connected in parallel. Whether this is a realistic description of the true effective coupling depends not only on the importance of incoherent inter-chain transfers, but also on the arrangement and alignment of the chains in the sample. This mesoscopic arrangement of the molecules is our next topic.

\section{Components of a mesoscopic model}

The mesoscopic model we shall describe links electronic and atomistic descriptions and the more standard continuum device modelling. It is essentially the model of Ramos and Stoneham 
(2000), Ramos et al (2001, 2002) and Stoneham and Ramos (2001). In the model, we need to define a structure, and rules for carrier motion and recombination. (A 'typical' realization of a conducting polymer from one of our mesoscopic models is shown in figure 1, and gives some idea of the complexity of the structures involved.)

The mesoscopic approach goes beyond standard analyses in several respects. First, it recognizes that texture can be important, and allows textures to be included systematically. Secondly, space charge can be treated as discrete, which has surprisingly significant effects. Thirdly, in transport, one can avoid the usual continuum assumption of isotropic carrier mobilities. Fourthly, one can recognize that carrier injection may be uniform over the electrode. In all these respects, we are recognizing that organic semiconductors should not be regarded as like silicon with modified parameters.

At the other extreme, the intra-molecular properties of the polymer molecules carry over to the mesoscopic model. Electron affinities, ionization potentials and carrier mobilities should be very similar in the solid state, at least after allowance has been made for the actual molecular configuration and for basic inter-molecular interactions. There will certainly be some new features: inter-molecular recombination; and carrier tunnelling from one molecule to another. The effective mobility may well be controlled by inter-molecular transitions, not by intramolecular carrier motion. The trapping of charge, the percolation in charge transport and the effects of other localized charges will all have consequences which must be approached at the mesoscopic level. In principle, it is possible to include chosen trapped or interface charge (Riess et al 2001) to tailor internal barriers.

Further results we describe are for a cell of density $0.43 \mathrm{~g} \mathrm{~cm}^{-3}, 5669$ chains (22 connected to each electrode; these particular chains are normal to the electrode), mean length 10 monomers and a distribution of lengths with $1 /\left(2 \sigma^{2}\right) \sim 0.1$, so the distribution has halfmaxima just above 7 and just below 13 monomer chains (figure 10). Carriers can be injected from the electrodes either as a pulse or continuously. The inter-molecular charge transport between neighbour chains occurs by a charge hopping process. The jump rate is assumed to be the product of the tunneling factors (Stoneham and Ramos 2001) and a detailed-balance factor falling off exponentially with the difference between the electron affinity (for hole transport) or the ionization potential (for electron transport) of neighbour chains.

For the continuous case, one electron and one hole were injected at each iteration. The iterations are, of course, a computer timescale, but would correspond to a physical timescale of about one iteration per 10 ps (Stoneham and Ramos 2001)). The model was run for 1800 iterations (so about $18 \mathrm{~ns}$ ). A typical transit time is about 60 iterations so, typically, there will be around 60 electrons (and 60 holes) in nearly 6000 chains. This charge density, 1/100 chains, is larger than those typical of some real systems (perhaps 1 in 100 000), although this is slightly compensated by the relatively low density of chains. However, the experimental values are by no means certain, and the model can be run with different parameters. The present runs could be regarded as for a system in which many of the chains would not transport carriers.

Our present results show that most intra-chain recombinations occur in chains which are parallel to the electrodes. This is partly a matter of dwell time, but the bias is not very strong, perhaps an extra $50 \%$ or so per chain. An excess of intra-chain recombinations is also found for very short or very long chains. The individual carrier motions are, of course, primarily in the direction of the applied field, but most carriers have transient motions against the applied field. One unexpected result from our earlier study (Stoneham and Ramos 2001) was that effective trapping (charge remaining for times long compared with the typical transit times) could occur even in the absence of traps (i.e., all strands having the same electron affinity or ionization potential). This effective trapping (which has been reported as Coulomb glass behaviour (Donovan et al 1993a, 1993b)) competes with radiative recombination. The individual carrier 


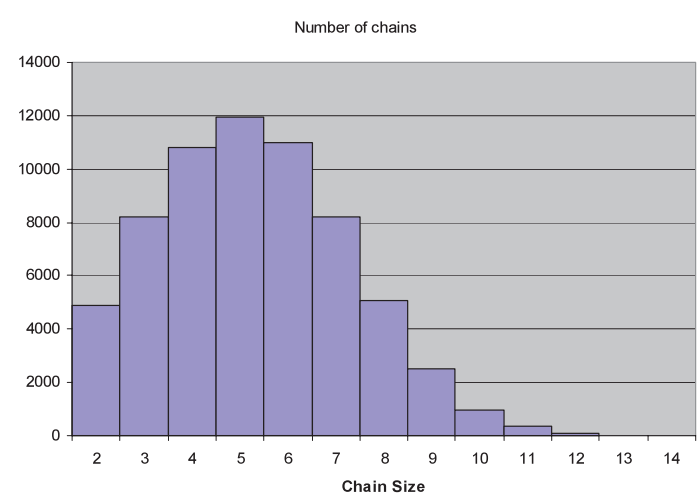

(a)

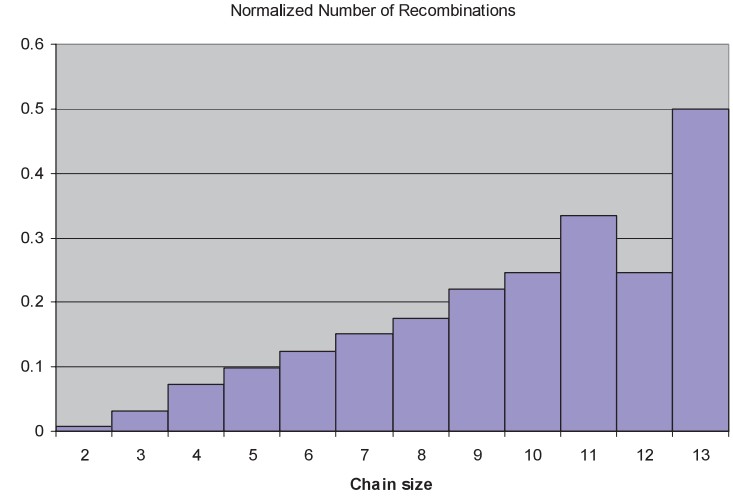

(b)

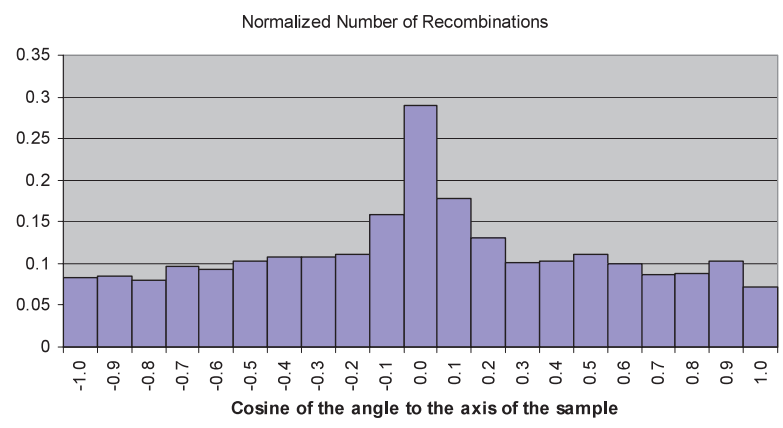

(c)

Figure 10. Results of mesoscopic modelling of carrier recombination events of the type discussed in the text. The panels show: (a) the distribution of chain lengths (measured in monomer units) in the ensemble; (b) the mean number of recombinations per chain during the simulation as a function of chain length; and (c) the mean number of recombination events per chain as a function of the chain's direction cosine along the direction of current flow.

(This figure is in colour only in the electronic version) 
motions are, of course, primarily in the direction of the applied field, but most carriers have transient motions against the applied field.

Further valuable information about the local electronic properties of polymer films and their coupling to light fields, which could be used as input for simulations such as these, could be gained by experiments using scanning probe techniques. For example, an STM tip can be produced both as a current source to produce local electroluminescence (Alvarado et al 2001) and for local electronic spectroscopy (Rinaldi et al 2001).

\section{Conclusions}

We have highlighted a number of areas in which further progress seems essential for a proper quantitative understanding of charge and energy transport in organic materials, especially in LEDs. These include: a better understanding of the transition from coherent to incoherent transport within a single chain; a more realistic treatment of charge injection from the electrodes, incorporating both realistic electrode structure and relaxation effects of the chains; a better understanding of the fundamental physics of inter-chain hopping (including the electronic matrix elements involved and the extent to which the process is a coherent one); and above all the nature of the disordered structure of the entangled chains and its effect on charge flow. Of the inter-chain effects, the least well understood is the process of interconversion between singlet and triplet excitons, which has important implications for the maximum attainable optical quantum yield from recombination.

Several scientific themes are likely to underlie future developments in these areas. First, it is necessary to marry the relatively sophisticated treatments that are possible for single chains on nanometre length scales with the materials science of the problem, for much of which longer length scales are relevant. Second, the effects of several kinds of local interaction on the motion of the charge carriers need to be better understood. These include the electron-electron and electron-phonon interactions (which result in the formation of composite entities such as the excitons and polarons referred to above). Third, these local interactions coexist with strong disorder (arising from the materials science issues mentioned above); furthermore, this disorder is almost certainly not of the pure 'hopping disorder' or 'site disorder' types beloved of theoreticians. Fourth, all of these factors must come together to control the effective parameters of 'drift-diffusion'-type models that can be used to model the behaviour of larger devices.

At the same time as these theoretical issues are being clarified, further experimental progress is being made on controlling molecular systems down to nanometre length scales. Resolving the theoretical questions will require continuing close collaboration between the conceptual and experimental efforts.

\section{Acknowledgments}

Part of this work was supported by the UK EPSRC. We would like to thank Dr Alison Walker and Dr Agapi Kimbali for a number of discussions of inter-molecular electron transfer in organic materials, and Graham Conroy for producing figure 1.

\section{References}

Almeida A M and Ramos M M D 2001 Synth. Met. 122165

Alvarado S F, Rossi L, Müller P, Seidler P F and Riess W 2001 IBM J. Res. Dev. 4589

Barth S, Wolg U, Bässler H, Müller P, Riel H, Vestweber H, Seidler P F and Riess W 1999 Phys. Rev. B 608791

Becker H, Burns S E and Friend R H 1997 Phys. Rev. B 561893

Blades C D J and Walker A B 2000 Synth. Met. 111-112 335-40 
Bloor D, Koski D, Stevens L, Preston G C and Ando F H 1975 J. Mater. Sci. 101678

Bolognesi A, Botta C, Fanchinetti D, Jandke M, Kreger K, Strohriegl P, Relini A, Rolandi R and Blumstengel S 2001 Adv. Mater. 131072

Bonca J and Trugman S A 1995 Phys. Rev. Lett. 752566

Burroughes J H, Bradley D C, Brown A R, Marks R N, Mackay K, Friend R H, Burn P L and Holmes A B 1990 Nature 347539

Chao K A and Wang Y 1985 J. Phys. C: Solid State Phys. 18 L1127

Conwell E M and Wu M W 1997 Appl. Phys. Lett. 701867

Cornil J, Beljonne D, Calbert J-P and Brédas J L 2001 Adv. Mater. 13 1053-67

Curioni A and Andreoni W 2000 Synth. Met. 111-112 299

Curioni A and Andreoni W 2001 IBM J. Res. Dev. 45101

Daubler T K, Glowacki I, Scherf U, Ulanski J, Horhold H H and Neher D 1999 J. Appl. Phys. 866915

Donovan K J, Elkins J W P and Wilson E G 1993a Synth. Met. 55-57 5032

Donovan K J, Elkins J W P and Wilson E G 1993b Mol. Cryst. Liq. Cryst. 236157

Friend R H, Gymer R W, Holmes A B, Burroughes J H, Marks R N, Taliani C, Bradley D D C, Dos Santos D A, Brédas J L, Lögdlung M and Salaneck W R 1999 Nature 397121

Gao Z Q, Lai W Y, Wong T C, Lee C S, Bello I and Lee S T 1999 Appl. Phys. Lett. 743269

Giebeler C, Whitelegg S A, Campbell A J, Liess M, Martin S J, Lane P A, Bradley D D C, Webster G and Burn P L 1999 Appl. Phys. Lett. 743714

Harrison M G, Ziemelis K E, Friend R H, Burn P L and Holmes A B 1993 Synth. Met. 55218

Heeger A J, Kivelson S, Schrieffer J R and Su W P 1988 Rev. Mod. Phys. 60781

Hochfilzer C, Leising C, Gao Y, Forsythe E and Tang C W 1998 Appl. Phys. Lett. 732254

Hoofman R J O M, de Haas M P, Siebbeles L D A and Warman J M 1998 Nature 39254

Itoh N and Stoneham A M 2001 Materials Modification by Electronic Excitation (Cambridge: Cambridge University Press)

Jarrett C P, Brown A R, Friend R H and de Leeuw D M 1995 J. Appl. Phys. 776289

Johansson A and Stafstrom S 2001 Phys. Rev. Lett. 863602

Kim Ji-Seon, Ho P K H, Greenham N C and Friend R H 2000 J. Appl. Phys. 881073

Kobrak M N and Bittner E R 2000 Phys. Rev. B 6211473

Lee J, Sundar V C, Heine J R, Bawendi M G and Jensen K F 2000 Adv. Mater. 121102

Magoga M and Joachim C 1999 Phys. Rev. 5916011

Ness H and Fisher A J 1999 Phys. Rev. Lett. 83452

Ness H and Fisher A J 2002a Europhys. Lett. 57885

Ness H and Fisher A J 2002b Chem. Phys. at press

Ness H, Shevlin S A and Fisher A J 2001 Phys. Rev. B 63125422

Newton R G 1960 J. Math. Phys. 1319

Nguyen T Q, Kwong R C, Thompson M E and Schwartz B J 2000 Appl. Phys. Lett. 762454

Pople J A and Beveridge D L 1970 Approximate Molecular Orbital Theory (New York: McGraw-Hill)

Ramos M M D and Almeida A M 2002 Vacuum 64 99-104

Ramos M M D, Almeida A M, Carneiro J P M and Stoneham A M 2001 Proc. CSC'4 (Paris: Société Française du Vide) pp 108-15

Ramos M M D, Almeida A M, Ramos M D and Cadilhe A M 2002 Comput. Mater. Sci. at press

Ramos M M D and Stoneham A M 1998 Proc. 3rd Int. Conf. on Electric Charge in Solid Insulators (Supplément à la Revue 'Le Vide: Science, Technique et Applications') No 287 ed G Damamme (Paris: Société Française du Vide) p 5

Ramos M M D and Stoneham A M 2000a Comput. Mater. Sci. 17260

Ramos M M D and Stoneham A M 2000b Synth. Met. at press

Ramos M M D, Stoneham A M and Sutton A P 1993 Acta Metall. Mater. 41 2105-11

Ramos M M D, Stoneham A M and Sutton A P 1994 Synth. Met. 67137

Riess W, Riel H, Beierlein T, Bruetting W, Mueller P and Seidler P F 2001 IBM J. Res. Dev. 45

Rinaldi R, Cingolani R, Jones K M, Baski A A, Morkoc H, Di Carlo A, Widany J, Della Sala E and Lugli P 2001 Phys. Rev. B 63075311

Salafsky J S 1999 Phys. Rev. B 5910885

Shi Y, Liu J and Yang Y 2000 J. Appl. Phys. 874254

Sixl H 1984 Polydiacetylene ed D Bloor (Amsterdam: Nijhoff) pp 240-5

Sols F 1992 Ann. Phys., NY 214386

Stoneham A M 1975 Theory of Defects in Solids (Oxford: Oxford University Press)

Stoneham A M 1981 Rep. Prog. Phys. 441251 
Stoneham A M 1991 Optical Properties of Excited States in Solids ed B di Bartolo (New York: Plenum) p 97 Stoneham A M 1998 Proc. 3rd Int. Conf. on Electric Charge in Solid Insulators (Supplément à la Revue 'Le Vide: Science, Technique et Applications') No 287 ed G Damamme (Paris: Société Française du Vide) p 1

Stoneham A M and Ramos M M D 1993 J. Solid State Chem. 106 2-12

Stoneham A M and Ramos M M D 2001 J. Phys.: Condens. Matter 132411

Stoneham A M, Ramos M M D and Sutton A P 1993 Phil. Mag. A 67 797-811

Tada K and Onoda M 2002 J. Phys. D: Appl. Phys. 35192

Tessler N, Denton and Friend R H 1996 Nature 382695

Tessler N, Harrison N T and Friend R H 1998 Adv. Mater. 1064

Wallace D S, Stoneham A M, Hayes W, Fisher A J and Harker A H 1991a J. Phys.: Condens. Matter 3 3879-904

Wallace D S, Stoneham A M, Hayes W, Fisher A J and Testa A 1991b J. Phys.: Condens. Matter 3 3905-20

Whitehead K S, Grell M, Bradley D D C, Jandke M and Strohriegl P 2000 Appl. Phys. Lett. 762946

Wilson J S, Chawdhury N, Al-Mandhary M R A, Younis M, Khan M S, Raithby P R, Kohler H and Friend R H 2001a J. Am. Chem. Soc. 1239412

Wilson J S, Dhoot A S, Seeley A J A B, Khan M S, Kohler A and Friend R H 2001b Nature 413828

Wohlgenannt M, Jiang X M, Vardeny Z V and Janssen R A 2002 Phys. Rev. Lett. 88197401

Wolf U, Arkhipov V I and Bässler H 1999 Phys. Rev. B 597507

Wu Guozhang et al 2000 J. Appl. Phys. 881480 